\title{
Spectral and global diffuse properties of high-performance translucent polymer sheets for energy efficient lighting and skylights
}

\author{
Geoffrey B. Smith, Jacob C. Jonsson, and James Franklin
}

\begin{abstract}
A visible and near-IR spectral study is presented for a translucent smooth polymer sheet in which dopant particles are clear polymer with a refractive index close to that of the clear polymer host. Diffuse, specular, and total reflectance and transmittance and absorptance as a function of sheet thickness and dopant levels approach ideal behavior for lighting applications. A fourth optical parameter, side loss $S_{T}$. is introduced to fully account for the measured data. This covers radiation that is trapped by total internal reflection (TIR) and travels sideways sufficiently far, including to the sheet's edges, to miss detection on exit. $S_{T}$ has a strong spectral character, whereas total $T$ and $R$ spectra closely follow the spectrally flat wavelength dependence of the undoped clear sheet. Three distinct regimes are identified for the behavior with wavelength of the specular and diffuse components and are linked to rear surface TIR and side loss. (O) 2003 Optical Society of America

OCIS codes: $\quad 160.4760,160.5470,290.5850,120.6200,120.7000$
\end{abstract}

\section{Introduction}

Translucent polymer sheets, and other sheets used in lighting and skylights, are traditionally pigmented with inorganic additives such as $\mathrm{CaCO}_{3}$, $\mathrm{TiO}_{2}$, and $\mathrm{BaSO}_{4}$. The particles' refractive index relative to the polymer host, their specific micrometerscale sizes, and the amount of additives combine to produce significant spreading of transmitted light. This spreading can be tailored as desired to reduce glare when the transmitting surface is viewed directly and to provide more uniform lighting when used in skylights or Luminaire covers. Traditional Mie-scattering theory modified to include multiplescattering effects is able to semiquantitatively describe their spectral transmittance and reflectance and light spreading. ${ }^{1}$

The underlying weakness of these traditional additives is that concentrations high enough to achieve

G. B. Smith (g.smith@uts.edu.au) and J. Franklin are with the Department of Applied Physics and the Institute of Nanoscale Technology, University of Technology, Sydney, P.O. Box 123, Broadway New South Wales 2007, Australia. J. C. Jonsson is with the Department of Materials Science. Lppsala University, P.O. Box 534, Uppsala SE 75121 , Sweden.

Received 15 September 2002; revised manuscript received 17 March 2003.

0003-6935/03/193981-11\$15.00/0

(c) 2003 Optical Society of America a reasonable spread of transmitted light intrinsically result in a significant degree of backscattering with consequent reduction of hemispherical transmittance. This is also why they look quite milky or creamy when viewed in reflectance. For example, we have characterized opal (PMMA) sheets as used for skylights and the pigmented polycarbonate widely used in roof glazing. We found that, at usual pigment levels, total transmittance of visible light can be down to near 50\%. For light fittings, the result is low light output ratios, which then requires larger lamp powers to achieve design illuminance. For skylights, a low hemispherical transmittance requires larger roof openings for a given interior illumination level. This in turn increases solar heat gain, which is undesirable in summer, and also increases heat loss in winter.

What characteristics should we require of ideal translucent material for lighting? We suggest the following: a tailorable light-spreading capability according to the additive concentration or sheet thickness combined with a high total visible transmittance that is independent of the degree of light spreading. This means that absorptance must be low. We also require low hemispherical visible reflectance under a wide range of conditions, although the admix of diffuse and specular reflectance may change. In addition, transmitted light in general should not be color shifted relative to the source light's color by the scat- 
tering process. To achieve all this with particle additives, the light-spreading process at each particle must lead to a high ratio of forward scattering to backscattering. In addition, the spread of light from any given direction upon striking a particle should be neither too large nor too sensitive to wavelength across the visible. A material approaching these characteristics has recently become available. The additive is itself a clear polymer particle and it is embedded in a clear polymer.

In this paper we address, mainly from an experimental viewpoint, the hemispherical and specular optical properties of smooth-surface PMMA sheets with different levels of clear polymer particle additives for four different sheet thicknesses. A number of interesting new optical features arise including the need to introduce side transmittance as a component in addition to reflectance, transmittance, and absorptance to completely define and understand optical measurements in these systems. We show that the presence of significant side transmittance means that standard measurements of hemispherical spectral transmittance significantly underestimate the transmittance of a uniformly illuminated sheet. This side-loss effect is a result of the combination of optical properties of the clear particles, their scattering pattern, low absorption in the sample, and the smooth exit surfaces. The extensive normalincidence experimental data needed to fully describe these effects, plus relevant luminance images, are sufficiently complex and interesting, but we will leave a detailed description and quantitative physical analysis of light spreading and angle of incidence effects to a later paper. However, the breakup of total spectral transmittance into diffuse and specular components presented here depends in an interesting way on wavelength. This is due to a combination of the spectral variation in the spread of transmitted light and the way this division is defined by experimental geometry. To aid in understanding this behavior and the spectral variations of side loss, a brief introduction to the underlying scattering models is given in Section 2. A preliminary report at one wavelength of light spread versus total transmittance in a limited but different set of polymer doped samples compared with some traditionally pigmented sheets has appeared previously. ${ }^{2}$

To provide a basis for understanding the distinct optical results that follow, a simple outline of how the central material and geometric properties influence forward transmission and backreflectance is given in Section 2. We then describe experimental measurements, present a range of spectra and some images, and analyze their unique features. We conclude with some implications for applications.

\section{Clear Polymer Microparticles in Clear Polymer: Optical Features}

Low loss, with almost negligible backscattering, can be achieved using a new type of transmission diffusing element, for which we have recently introduced ${ }^{3}$ the transparent refractive-index matched micropar- ticle (TRIMM) diffuser, to describe one particular application. The acronym TRIMM embodies the key physical features of the particle and matrix, namely, (i) each additive particle is several micrometers in diameter (preferably over $6 \mu \mathrm{m}$, and we have successfully used up to $35 \mu \mathrm{m}$ ), (ii) the particle does not absorb visible light and hence is visibly clear and transparent if smooth, and (iii) its refractive index is close to that of the host polymer. It is also of course desirable that the host polymer be highly transparent at visible wavelengths.

van de Hulst ${ }^{4}$ devotes a chapter to a detailed treatment of the large sphere (relative to wavelength) limit of Mie scattering. It is shown that in this limit one can either use ray optics or equivalently take the asymptotic limit of the Mie-scattering equations. A key parameter in these models is the refractive-index ratio $m=n_{\text {particle }} / n_{\text {host }}=1+\mu$, which allows one to write the difference $n_{\text {host }}-n_{\text {particle }}=\mu n_{\text {host }}$, where $\mu$ can be positive or negative. The energy efficiency for lighting applications of these doped polymers, and the other novel features that we detail below, arise primarily because each sphere gives negligible backreflectance. This occurs because the refractive index of the embedded spheres is quite close to that of the matrix, so $\mu$ is a small number. As an example, we determined, both by using an Abbe refractometer with the sample in refractive-index matching liquid and by fitting scattering data with models, that at $633 \mathrm{~nm}$ in two of the samples studied below, $\mu=$ 0.011 . As a ray strikes the surface of a sphere, standard Fresnel equations can be used to determine its probability of reflection and transmission. Fresnel reflectance is quite weak, especially for the $50 \%$ of rays in a parallel beam that can be backreflected as they strike a sphere surface at less than a $45^{\circ}$ incidence angle. Their probability of backreflectance is close to $(\mu / 2)^{2}$, which is approximately equal to $0.003 \%$. Only glancing rays are significantly forward reflected but they make up an almost negligible fraction of total flux onto a sphere. ${ }^{4}$ The approach to the geometrical-optics limit can be seen in the singlesphere scattering patterns presented by Sorensen and Fischbach ${ }^{5}$ and is accelerated by any slight variations in particle size ${ }^{4}$ or shape, plus multiple-sphere interactions, which each wash out residual singlesphere phase-related oscillations. These influences are all present in our samples.

To simulate the transmittance pattern, multiple ray tracing is used, with each ray encountering several spheres. The deviation of any ray at a sphere of radius $R$ can be simulated from either a single ray analysis or from the Mie result. Since $\mu$ is so small, all rays that enter the sphere are assumed to exit the first time they strike the surface. The resultant deviation angle $\delta$, as shown in Fig. 1(a), of the exit ray from the incident direction is given by

$$
\delta=2\left\{\sin ^{-1}(h / R)-\sin ^{-1}[h / R(1+\mu)]\right\},
$$

where $h$ is the perpendicular separation of the ray from the parallel ray that passes through the sphere's 
(a)

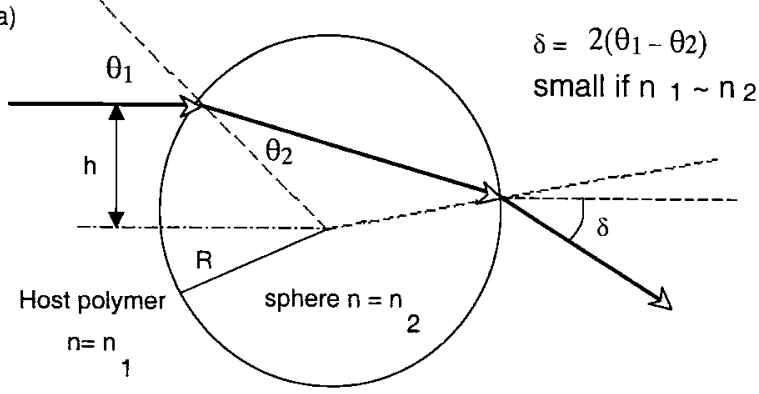

(b)

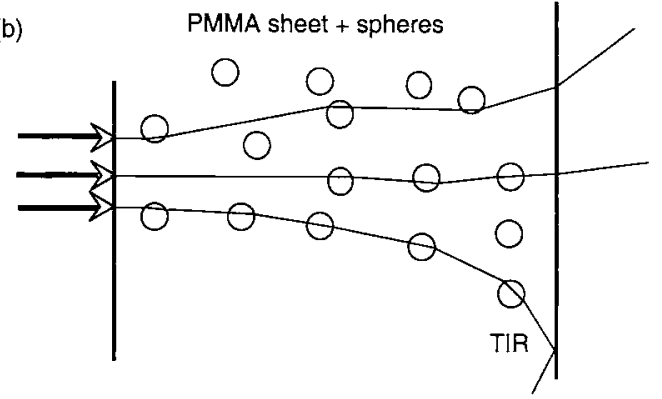

Fig. 1. (a) Definition of different parameters when a single ray enters a sphere. The deviation angle is heavily magnified compared with that in the materials discussed in this paper. (b) Schematic of rays traversing a clear sheet doped with clear spheres including one ray that is totally internally reflected.

center. It is noteworthy that $\delta$ depends only on $h / R$ and $\mu$. This means that (in the geometric limit) the single-particle scattering pattern is completely independent of the particle size distribution-it depends only on $\mu$. Accordingly, any variation in the scattering pattern that is wavelength dependent follows solely from changes in $\mu$ with respect to wavelength. Note that small changes in the absolute refractive index of particle or host can give rise to significant changes in $\mu$, and hence in the particle's scattering properties. For constancy in scattering properties, we require a close match in the host and particle's dispersion properties over the wavelength region of interest.

Since $\mu$ is small, $\delta$ in Eq. (1) is clearly small, except for the few rays that enter at near-glancing incidence. For deviation due to rays striking two or more spheres, changes in the azimuth angle ( $\phi)$ and hence the plane of incidence relative to that of the first sphere must also be allowed. Details on how to include this will appear in reports on our angle-resolved studies not included in this paper. For multiple spheres, the average distance traveled between spheres is needed, and this is easily calculated from concentration or can be measured directly. For a given particle-host polymer combination with a fixed $\mu$ value, light spreading is thus tailored simply by varying the number of spheres encountered by changing the sphere concentration or sheet thickness.

An alternative approach for a single sphere is to work with the scattered intensity distribution function $i(\delta)$ for a single sphere in the limit of small $\mu$ and a large sphere radius. ${ }^{4}$ As given by Eq. (2), it is expressed in terms of the peak value $i(0)$ measured at $\delta=0^{\circ}$ where $i(0)$ is inversely proportional to $\mu^{2}$.

$$
i(\delta)=i(0)\left[\frac{1}{1+\left(\delta^{2} / 4 \mu^{2}\right)}\right]^{2} .
$$

Since $\mu$ is small, it is clear from Eq. (2) that $i(\delta)$ is also small for $\delta \gg \mu$. Thus the spread of exit angles from one sphere for parallel incidence will be confined to a small range of deviation angles $\delta$, and interactions with several spheres will be needed to achieve a significant spread of exit angles, even allowing for the additional deviation that occurs at a sheet's exit surface due to Snell's law.

Another feature of having a small $\mu$ and large spheres relative to wavelength is that the same forward-scattering pattern emerges for $m$ values that are less than or greater than 1 by the same small amount (i.e., at the same $\mu$ magnitude but at opposite signs). ${ }^{4}$ Equivalently, at low enough $\mu$, replacing the $m$ ratio by $1 / m$ gives the same pattern. That is, we expect similar light-spreading patterns whether the host polymer has a larger or smaller index than the embedded sphere and the models apply for either sign of $\mu$. The refractive index of PMMA, the host in this study, falls slightly as wavelength increases from its value of 1.496 near $400 \mathrm{~nm}$ to its value of 1.488 near $700 \mathrm{~nm}$, and hence $m$ and $\mu$ are also expected to vary with wavelength. Since the transmitted light distribution is quite sensitive to the magnitude of $\mu$, we thus expect a wavelength dependence in these scattering patterns. The spectral dependence of the breakup of hemispherical transmittance into diffuse and specular transmittance, one of the key results that we report below, is one consequence of the spectral dependence of $\mu(\lambda)$. Color effects for a broadband incident spectrum are another. Achieving minimal color variations across the exit beam with a polychromatic incident beam requires that $\mu(\lambda)$ be reasonably constant over the spectral range of interest. The scattering angle $\delta$ for a single sphere at which the intensity is one half of the peak intensity is a good guide as to how overall spreading depends on $\mu(\lambda)$. This half-angle is, from Eq. (2), equal to $1.29 \mu$ in the small $\mu$ limit. Note, however, that colorrelated shifts are more apparent with a narrow collimated beam, as in the experiments here, than with broad-area illumination where any sampling point some distance from the sample receives radiation covering a wide range of scattering angles.

The net result of using TRIMM particles is high forward transmittance with an overall spreading of light that for a given wavelength depends on the total number of particles encountered on average by light traversing the sheet. This is combined with low backscattering even for a large number of encounters. A schematic covering these key features for a few rays appears in Fig. 1 (b). The deviation for any one ray is almost always small. It changes for rays 
striking different points on the sphere, and only the few rays striking at grazing incidence undergo angular deviations more than a few degrees. The data that follow for normal-incidence illumination of sheets at three different particle concentrations, each at four thicknesses, clearly show the results of these small deviations and negligible reflectance. The total hemispherical reflectance $\left(R_{h}\right)$ never rises over 0.10 , which is just 0.023 above that for a smooth undoped PMMA sheet. We show that the small increment in $R_{h}$ above the value for a smooth transparent sheet is largely attributable to the effect of the smooth exit surface. This effect is quite novel and probably specific to this type of high-transmittance translucent system. The exit surface has an even more significant and fundamental affect on the combined measured optical data, which necessitates that we introduce a new optical parameter in addition to the usual three parameters that describe the transport of light energy after striking a material, namely, reflectance $R$, transmittance $T$, and absorptance $A$. We thus need to analyze the exit surface impact further to define this property before presenting the data.

Light rays striking the smooth exit surface will be distributed over a range of directions, some of which may have an angle of incidence above $\theta_{\text {crit }}$, the critical angle for total internal reflection (TIR), and thus cannot exit the sheet. The internal intensity pattern at this surface $I_{\mathrm{in}}(\theta, \phi)$ is generally independent of the azimuth angle $\phi$. Thus, after reduction by the reflectance coefficient at each angle by use of the factor $[1-R(\theta)]$ with $R=1$ for $\theta>\theta_{\text {crit }}$ and modification as a result of Snell's law, the emergent intensity pattern $I_{\text {out }}(\theta)$ can be found. Ray tracing leads directly to $I_{\text {out }}(\theta)$ from the emergent ray distribution scaled to a solid angle. Internally, the ray tracing leads to an internal density of rays as a function of $\theta$, which peaks at a finite $\theta$ value in the vicinity of the average deviation angle. A ray-tracing simulation study of one such distribution for transport in a polymerdoped polymer light guide has already been published ${ }^{3}$ and shows a distinct peak at a $\theta$ value, which is a function of length and particle concentration. A similar normal-incidence simulation for no edges (effectively a thin sheet with surface dimensions many times the thickness) produces a peak in ray density well away from the normal at an angle determined by concentration and thickness. Intensity still peaks at the normal when these ray densities are scaled to the relevant element of a solid angle, as verified by intensity measurements in a photogoniometer. As noted above, measurement and modeling of ray profiles in detail will be left to a future paper. The spectrum of total, diffuse, specular, and side-loss $T$ and $R$ components is the focus here. Side loss arises from the rays that are totally internally reflected.

Many of those rays that do not escape because of TIR at the exit surface can, because of the special forward-only transport properties of these systems, continue to travel toward the edges of the sheet for quite a distance before finally escaping from the front

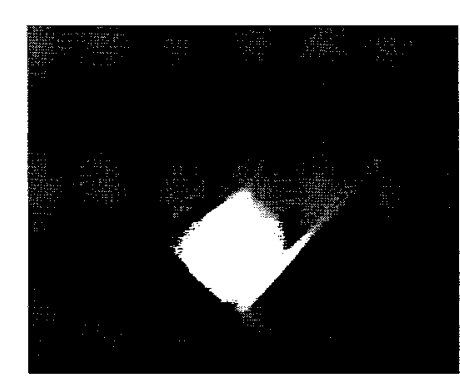

(a)

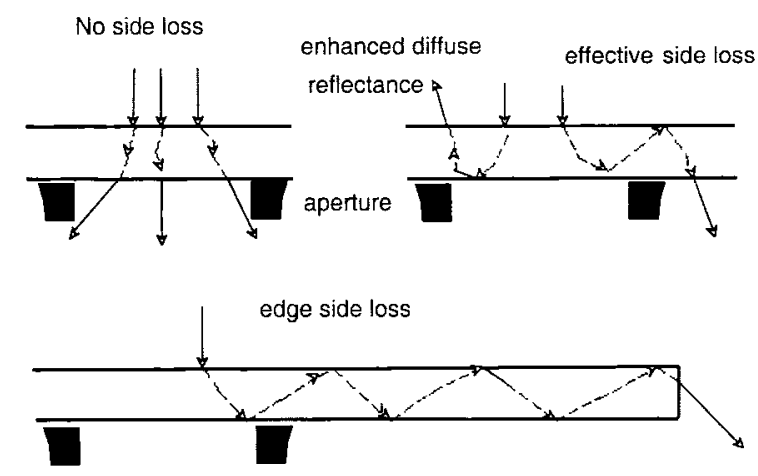

(b)

Fig. 2. (a) View of a doped sheet obliquely illuminated with a narrow beam. Both rays that transmit and exit after a single pass and those that make up the side-loss component can be seen. (b) Schematic of some different categories of ray paths in TRIMMdoped systems according to their contribution to measured components.

or back surface or even the sheet edges. Both of these effects can be seen in actual images in Fig. 2(a). In this image, a substantial fraction of the light is trapped inside the sheet and transported sideways to regions well removed from those studied in ordinary measurement instruments. Figure 2(b) shows schematically these various categories of ray paths relevant to what is shown in Fig. 2(a). Effective side loss can thus be seen to include rays that are not detected because of the limited size of the instrument's measurement ports. These rays eventually exit the edge or top or bottom surfaces outside the instrument's entrance port. Because of the finite apertures in the two sphere spectrometers used in this study (and in most spectrophotometers and integrating spheres), all side-loss light in general will not be recorded in either $T$ or $R$ measurements. Not only the port size, but also the shape of the illuminating beam, as shown in Fig. 3, affects the measured transmittance and reflectance values. Hence it is inappropriate to use the traditional definition of absorptance as $A=1-$ $T-R$, since absorbed light is converted to heat, whereas most of this lost or undetected light eventually exits. We thus introduce a new quantity called $S_{T}$ for side-transmitted light or "side-loss" light that is not recorded in the spectral measurement but is not absorbed. Then we can define

$$
A=1-T-R-S_{T}
$$




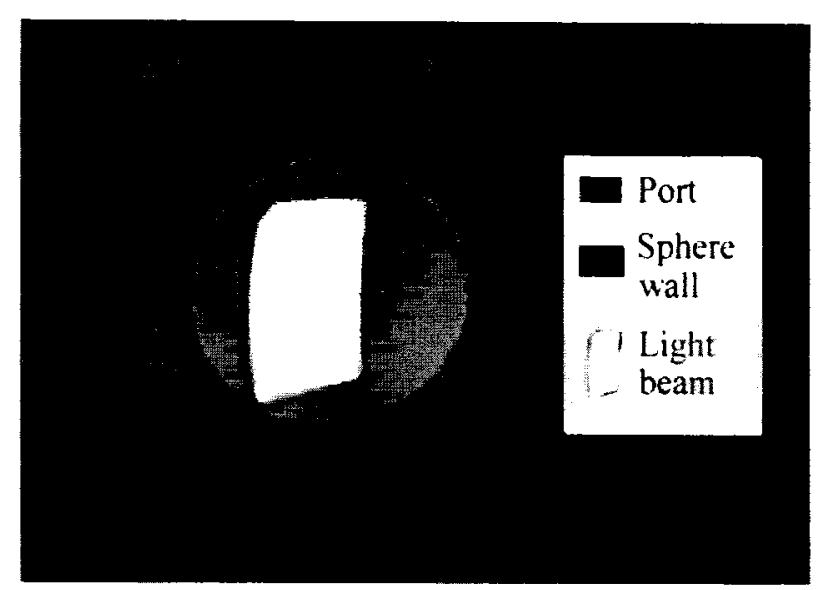

Fig. 3. Image of the sample illumination at the entrance port of the integrating sphere. Different parts of the beam will be detected differently because the mean path length to the port edge varies. Any light that misses the port gives rise to effective side loss because of the strongly forward-scattering nature of the sample studies here; the loss at the transmittance port is more crucial.

as the usual absorptance. The extinction coefficient or the imaginary part of the refractive index $k$ in undoped PMMA is less than $1 \times 10^{-6}$ (Ref. 6) from 400 to $750 \mathrm{~nm}$. Thus, in PMMA sheet systems of thicknesses up to $4 \mathrm{~mm}, A$ is small or negligible over the visible range. PMMA does have weak absorption due to its UV absorption tail plus some weak intrinsic bands near 600 and $700 \mathrm{~nm}$ and stronger peaks as the wavelength increases into the near infrared (NIR). Details on these absorption spectra in thick PMMA samples including polymer optical fibers can be found in Lekishivili et al. ${ }^{\bar{T}}$ For the visible wavelengths of interest in lighting and for thicknesses up to $4 \mathrm{~mm}$, we can accurately approximate $A=0$ and hence calculate the $S_{T}$ component from Eq. (3). Our data support this because, in some thicknesses and low-concentration combinations, the sum of $S_{T}$ and $A$ in Eq. (3) is too small to record whereas adding more PMMA spheres causes $A+S_{T}$ in Eq. (3) to become significant. Because there is no mechanism in the spheres for raising $A$ at these wavelengths, all additional loss should be due to side transport.

\section{Samples, Optical Measurements, and Results}

The sheets that we now study in detail consist of cross-linked PMMA spheres embedded in clear PMMA. Two of the sheet types are closely related optically to sheets that can be produced with commercially available molding beads with the generic name Plexiglas diffuser molding compound, manufactured by Roehm Degussa. Currently available diffuser materials are designated by the prefixes df20, df21, df22, df 23 , which represent successively higher concentrations of spheres. The optical properties of the resulting materials are determined by these four categories and sheet thickness. The type of clear molding beads with which the sphere-doped molding beads are mixed does not alter optical effects significantly but links to the mechanical and thermal properties. These different matrix materials are designated $7 \mathrm{H}, 7 \mathrm{~N}, 8 \mathrm{~N}$, and $\mathrm{z} 6$.

The clear-sphere-doped PMMA samples in this paper were formed by injection molding with a single mold containing four uniform thickness segments, 1 , 2,3 , and $4 \mathrm{~mm}$, and optically smooth surfaces. These samples were prepared by Roehm Degussa in Darmstadt, Germany. Three different mold mixes were used. Two used identical optical-grade clear PMMA molding beads (type $7 \mathrm{~N}$ ) mixed with $3 \%$ and $7 \%$ by weight of the same Plexiglas molding beads (1011F) that are doped with clear transparent crosslinked PMMA spheres with an average diameter of $15 \mu \mathrm{m}$. These two, which we label N73 $(3 \%)$ and N77 (7\%), have predominantly diffuse transmittance. They are designed to have optical properties similar to df22 and df23 grades but with less diffuser beads, which allows the final product to maintain the mechanical and rheological properties of the matrix polymer.

A third class of sheet used a different type of cleargrade PMMA molding beads, $8 \mathrm{~N}$, mixed with molding beads of type OV137. Because of the low concentration of scattering beads, this mixture is designated N80. This is a distinctly different product compared with the N73 and N77 samples. Sheets from the N80 material give different optical results to the other two diffuser materials noted above and are aimed at applications in which the sheet's edges are illuminated and light is emitted from the main surface. They have in common with the other samples a high total transmittance and are thus also energy efficient. They combine a substantial specular transmittance with a significant diffuse transmittance component that increases with thickness. Distinguishing features include a much lower density of particles, smaller spheres (typically 6 or $7 \mu \mathrm{m}$ across), and larger $\mu$. The latter is to compensate for the lower chance of a sphere encounter and to achieve the larger average shift in the deviation $\delta$ of Eq. (1) needed to provide a clearly visible display when viewed near the normal to the surface. The small $\mu$ approximation of Eq. (2) is less accurate but still useful for this system because reflection at each sphere is stronger, although it remains small. We can observe the spheres in these samples in reflectance mode on an optical microscope, whereas for N73 and N77 we can use only the transmission mode to image the spheres. Equation (1) is still accurate with this system.

The samples studied thus give a good coverage of the capabilities of these materials. Because of their cross linking, the PMMA spheres do not melt despite melting of the PMMA molding beads during the molding process. However, they may soften as evidenced by the thinner samples of N73 and N77 where many spheres are off spherical. This is presumably because of larger shear forces and more impact against the mold walls experienced in thin sections during the injection molding process. In the thicker samples, sphericity is largely maintained. Although 
quantitative details change with nonspherical shapes, the fundamental behavior (low $R$, small forward scattering per particle) discussed in Section 2 will be well maintained.

We obtained optical hemispherical and specular $T$ and $R$ data using a Perkin-Elmer Lambda 900 spectrophotometer at visible and NIR wavelengths. The spectrophotometer is equipped with the standard Labsphere 150-mm-radius Spectralon integrating sphere (PELA-1000). The angle of incidence is $8^{\circ}$ in the reflectance mode and normal in the transmittance mode. The sphere entrance ports are circular with a diameter of 20 and $25 \mathrm{~mm}$ for transmittance and reflectance, respectively. An image of the entrance port to the integrating sphere appears in Fig. 3 . The incident beam is smaller than this sphere's entrance port. Transmitted light that is unable to enter this port because it has traveled too far to one side owing to TIR [Fig. 2(b)] before finally emerging and the light emerging at the sample edges both contribute to side loss. The exit ports that are open for measuring the diffuse components are a $30 \mathrm{~mm} \times 30$ $\mathrm{mm}$ for reflectance and a 25 -mm-diameter circle for transmittance. This means that rays with deviations from the incidence direction of up to $4.8^{\circ}$ in transmittance and $6.7^{\circ}$ in reflectance are categorized as specular in the Lambda 900 spectrophotometer. These values are typical for this class of commercial integrating sphere spectrophotometers. The Spectralon coating of the sphere limits the instrument's effective wavelength region to $200 \mathrm{~nm}$ in the UV region and $2550 \mathrm{~nm}$ in the NIR where noise is quite significant. The absorbing characteristics of the PMMA make it unnecessary to investigate further down than $320 \mathrm{~nm}$ in the UV region. The noise in the lead sulphide detector used in the NIR region is approximately 0.01 . This uncertainty is absolute and hence is the same for all measurements. The photomultiplier tube, however, shows a relative noise contribution of the same order $(0.01)$ that results in a larger absolute uncertainty for the transmittance measurements.

The repeatability of the measurements was excellent and was independent of which side of the sample was illuminated. This confirms that the scattering spheres are homogeneously distributed. Note that, in practice, the admix of diffuse and specular transmittance and reflectance depends on the geometry of the integrating sphere and its apertures.

In principle we have a method to calculate the specular transmittance from the microstructure in these materials. The probability of no deviation of a normal-incidence ray is the probability of going straight through without striking even one sphere. This is given by $P(0)=\exp (-\langle p\rangle)$, where $\langle p\rangle$ is the average number of spheres encountered by any line traversing the sheet normal to the surface. For a given thickness, knowing $\langle p\rangle$ also gives the average path distance between sphere encounters. There is a direct relation between the probability of no deviation and the true specular transmittance for the sample, which is defined as light transmitted at no

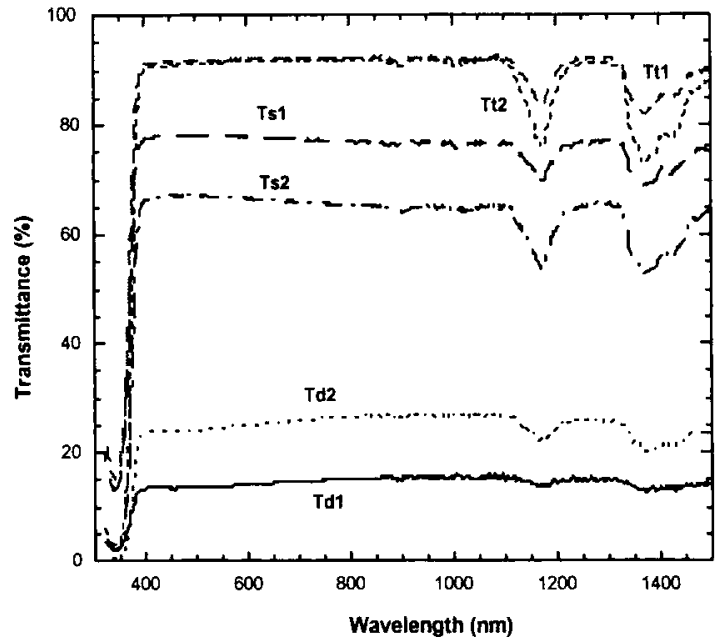

(a)

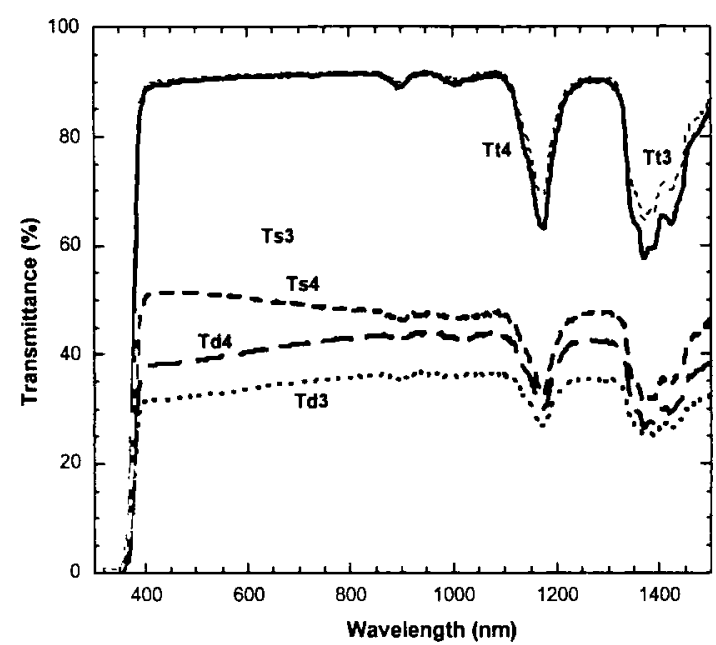

(b)

Fig. 4. Hemispherical total (Tt), diffuse (Td), and specular (Ts) transmittance spectral components for sample N80 from 300 to $1400 \mathrm{~nm}$ : (a) 1 and $2 \mathrm{~mm}$ thick, (b) 3 and $4 \mathrm{~mm}$ thick.

deviation. The resulting knowledge of sphere densities predicts, as might be expected, a lower specular $T$ value than we measure with integrating spheres and spectrophotometers. This is because, in standard measurement practice, specular incorporates an angular spread of a few degrees. Measurement of the transmitted light with a deviation of less than $0.1^{\circ}$ was used to determine the average distance between spheres. For this paper we retain the total instrument value with an acceptance angle of $4.8^{\circ}$.

Figures 4, 5, and 6 show how the specular and diffuse components of $T(\lambda)$ along with their sum (the total hemispherical transmittance) evolve. The changes follow the four thicknesses $(1,2,3$, and 4 $\mathrm{mm}$ ) and sphere doping levels, which are differentiated by sample types N80, N73, and N77, respectively. Figure 7 gives all spectral reflectance $R(\lambda)$ components for the N80 samples. The side-loss $S_{T}(\lambda)$ spectra appear in Figs. 8(a)-8(c) for the three sample classes, respectively, at each of the four thick- 


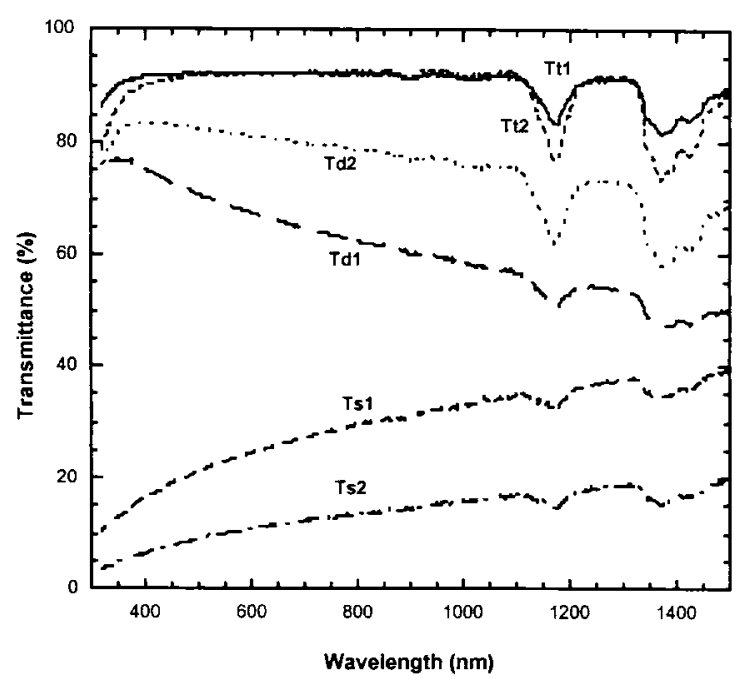

(a)

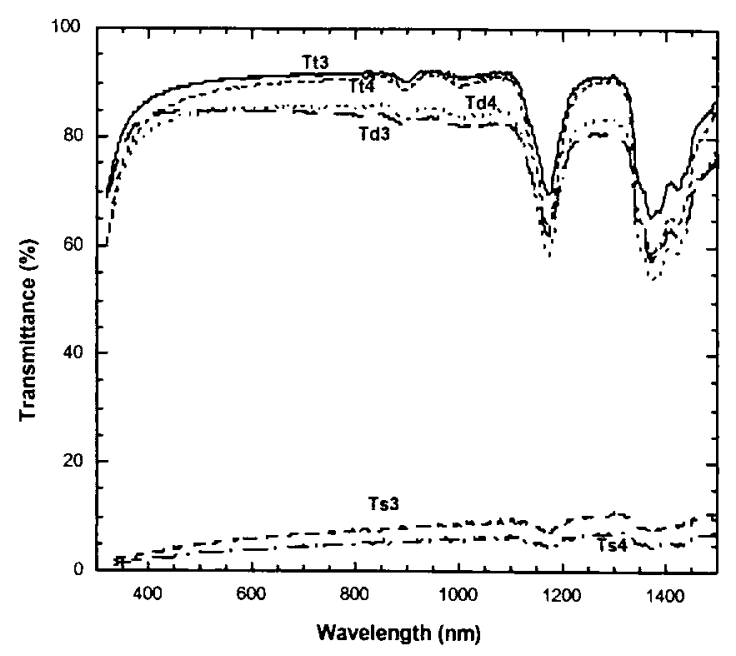

(b)

Fig. 5. Hemispherical total $(T t)$, diffuse $(T d)$, and specular $(T s)$ transmittance spectral components for sample N73 from 300 to $1400 \mathrm{~nm}$ : (a) 1 and $2 \mathrm{~mm}$ thick, (b) 3 and $4 \mathrm{~mm}$ thick.

nesses. Side-loss values under $2 \%$ display some noise, as do reflectance values for wavelengths beyond $900 \mathrm{~nm}$. It is also instructive to observe directly how particular spectrophotometric components vary with thickness at a fixed wavelength in the $400-$ $700-\mathrm{nm}$ range. We choose a wavelength of $520 \mathrm{~nm}$ and in Fig. 9 show the separate $T(\lambda)$ components and the total for the three samples. Figure 10 has the $R(\lambda)$ components and total $R$ for the three samples, and Fig. 11 combines the three side-loss versus thickness terms onto one plot to demonstrate the strong sensitivity of this term to the number of spheres encountered.

\section{Discussion}

The spectra and other plots in Section 3 have a number of interesting new features that link to these systems' abilities to combine energy efficiency with low glare and also to the control of the admix of

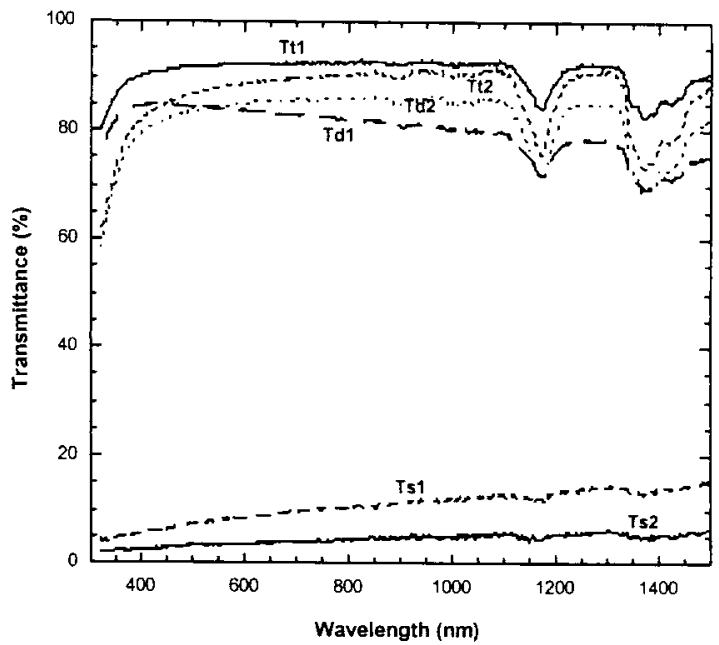

(a)

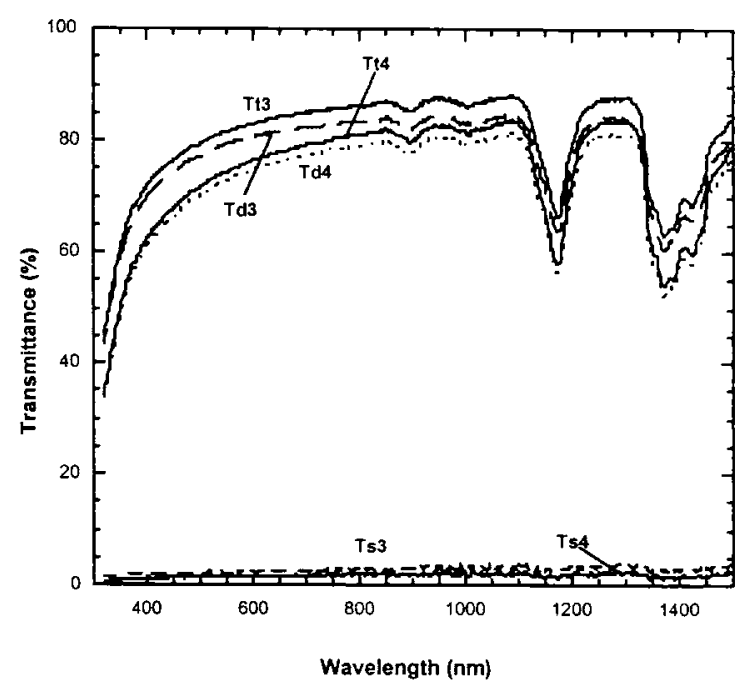

(b)

Fig. 6. Hemispherical total $(T t)$, diffuse $(T d)$, and specular $(T s \mid$ transmittance spectral components for sample N77 from 300 to $1400 \mathrm{~nm}$ : (a) 1 and $2 \mathrm{~mm}$ thick, (b) 3 and $4 \mathrm{~mm}$ thick.

specular and diffuse transmittance over a wide range.

One remarkable feature is the relative constancy of total $R(\lambda)$ and total $T(\lambda)$ in the visible range for all samples, despite wide spectral variations in the diffuse and specular component $R$ and $T$ values. In all but the most diffusing samples (the heavily doped 3 and 4-mm-thick samples of type N77), total hemispherical $T(\lambda)$ is between $91 \%$ and $94 \%$ and total $R(\lambda)$ is under $9 \%$. In other words, these numbers and spectral characters are remarkably close to the values of clear sheets. These total $T$ and $R$ spectra are also remarkably flat for a transmitting diffuser material with only the two most diffuse materials showing much variation with $\lambda$ over the visible range. Any spectral shifts and the changes in total $T$ and $R$ seem to be exactly correlated with the spectral changes in the side-loss terms shown in Fig. 8. This side-loss term is why total $T$ does shift noticeably 


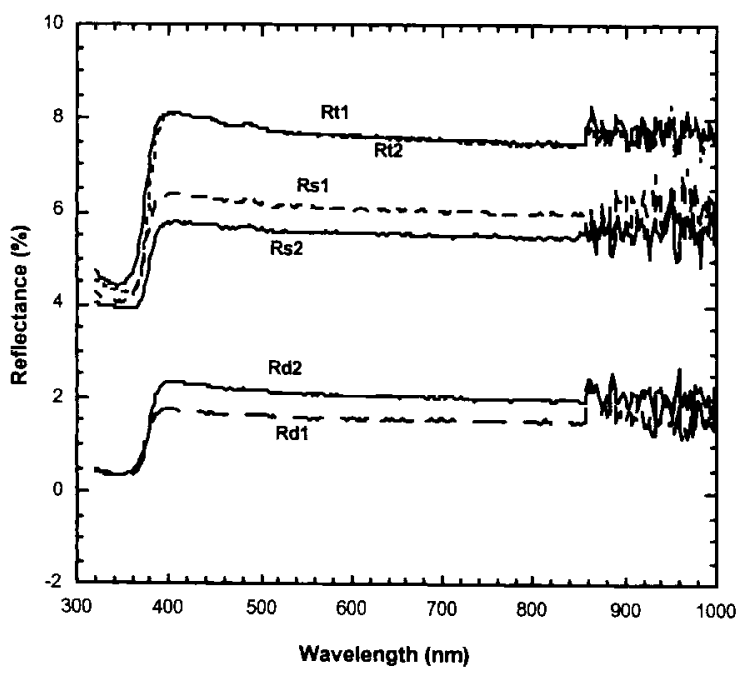

(a)

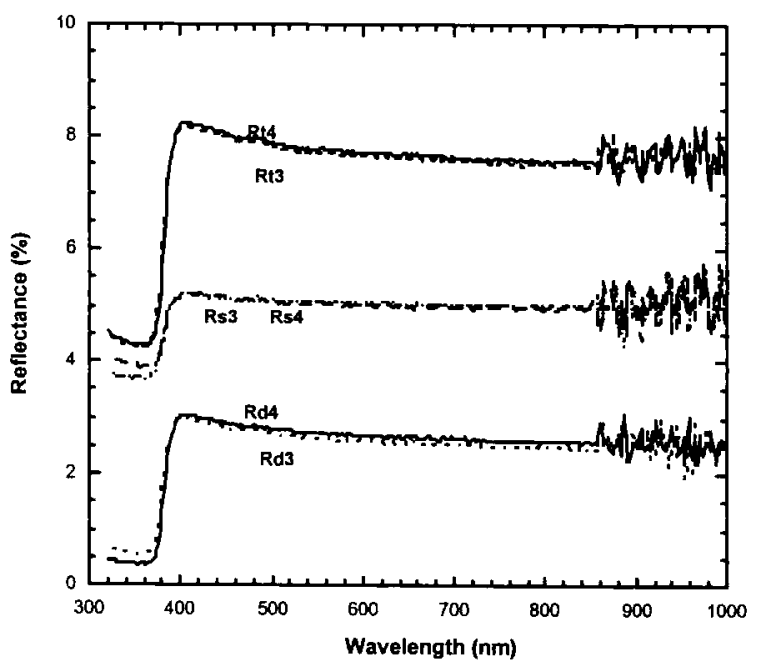

(b)

Fig. 7. Hemispherical total (Rt), diffuse $(R d)$, and specular $(R s)$ reflectance spectral components for sample N80 from 300 to 1000 $\mathrm{nm}$ : (a) 1 and $2 \mathrm{~mm}$ thick, (b) 3 and $4 \mathrm{~mm}$ thick.

with wavelength in the N77 case, where $S_{T}$ changes from $10 \%$ to $25 \%$ and $5 \%$ to $15 \%$ over the visible spectrum, in 4- and 3-mm-thick samples, respectively. In all other samples, the total change in $S_{T}$ across the visible is under $3 \%$. Most of this side loss is propagated sufficiently far so as to not contribute to either $T$ or $R$ readings as exemplified in Fig. 2. Most importantly, the physical process behind any spectral variations is not to be found in traditional particlescattering mechanisms that increase in strength as the wavelength shortens toward the blue. Instead, there is a diversity of spectral responses in these unusual materials, which we now examine more carefully.

The increase in nominal side loss seen around 900 $\mathrm{nm}$ for all three samples is possibly the result of absorption in the PMMA matrix. If $n_{\text {particle }}$ and $n_{\text {host }}$ are both changed, then it is quite likely that the spectral variation of their ratio may also change.

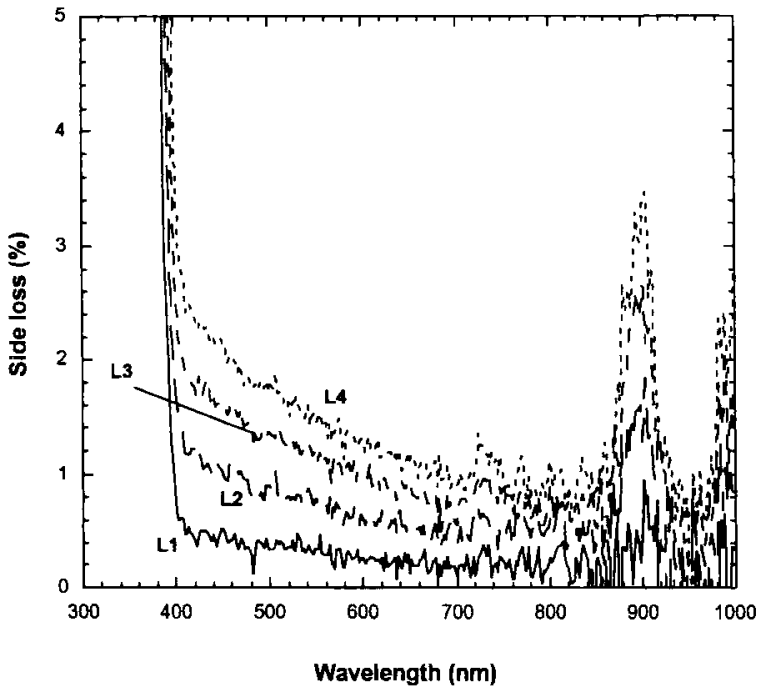

(a)

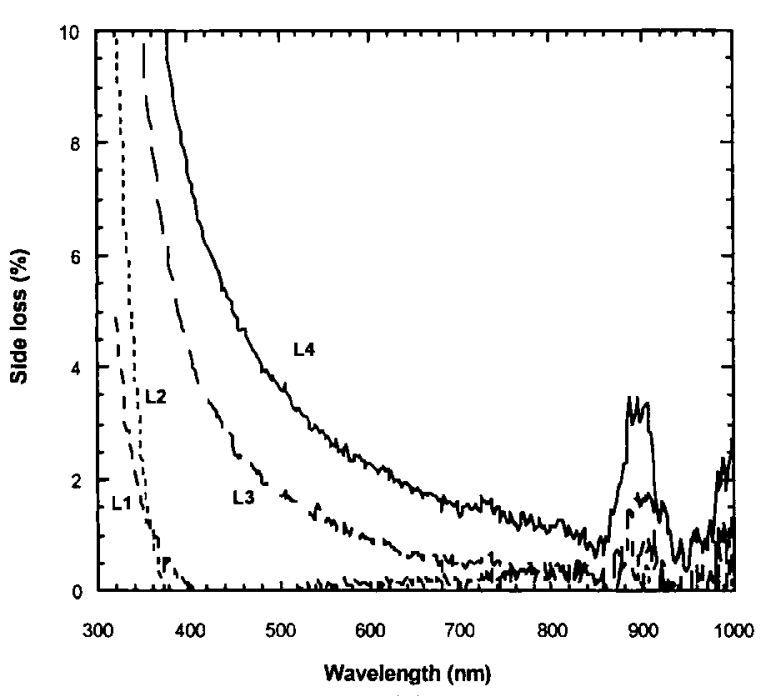

(b)

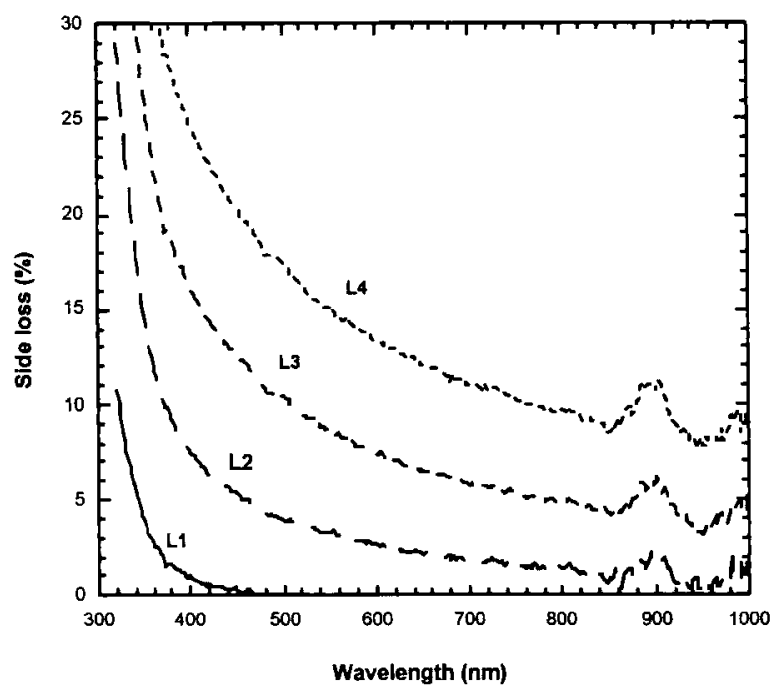

(c)

Fig. 8. Side-loss $S_{T}(\lambda)$ spectra from 300 to $1000 \mathrm{~nm}$ at 1-, 2-, 3-, and 4-mm thickness for sample classes: (a) N80, (b) N73, (c) N77. 


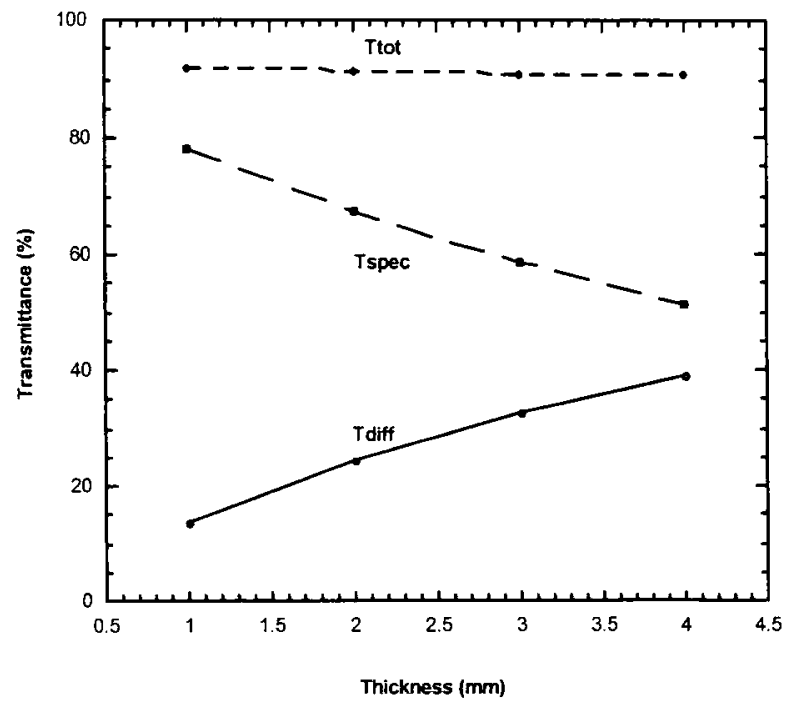

(a)

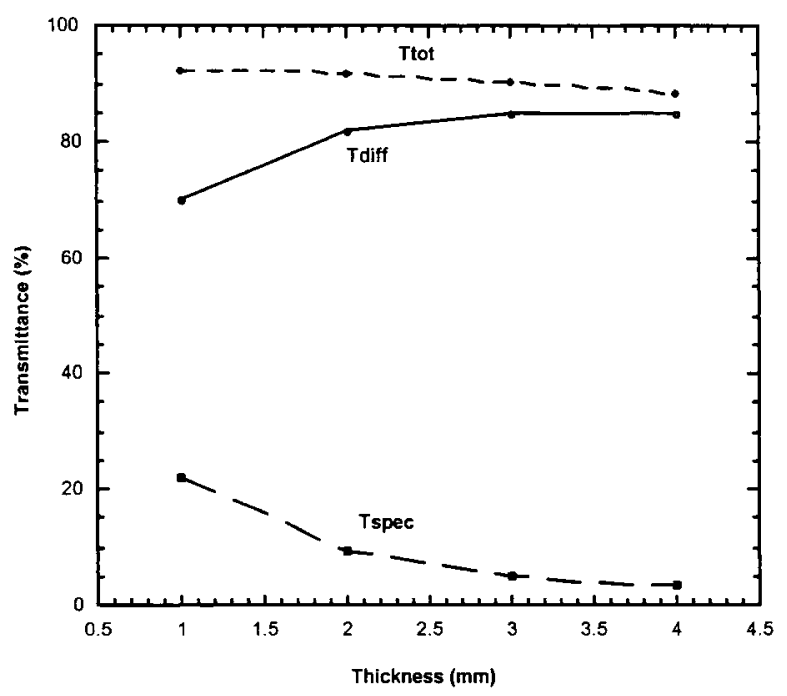

(b)

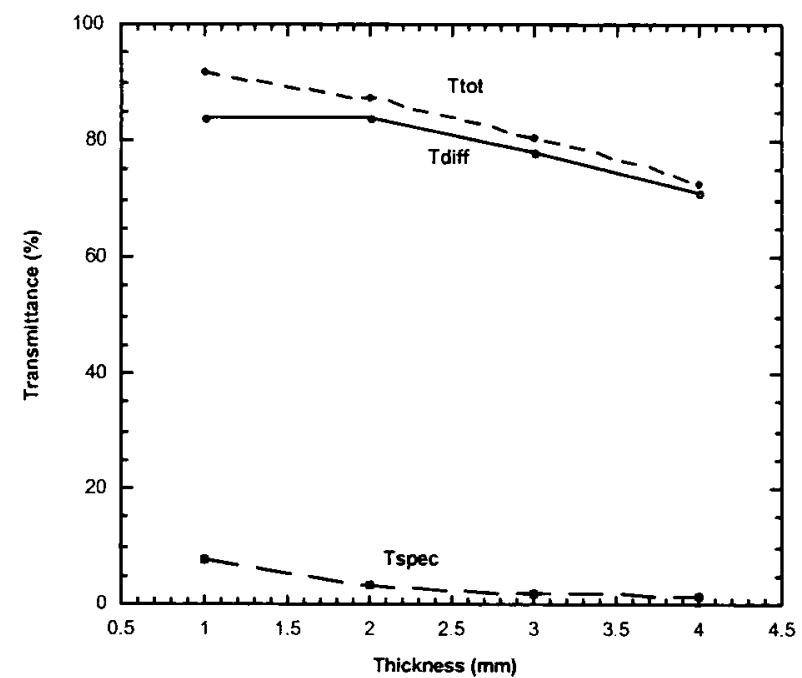

(c)

Fig. 9. Hemispherical total, diffuse, and specular transmittance at $520 \mathrm{~nm}$ as a function of thickness: (a) N80, (b) N73, (c) N77.

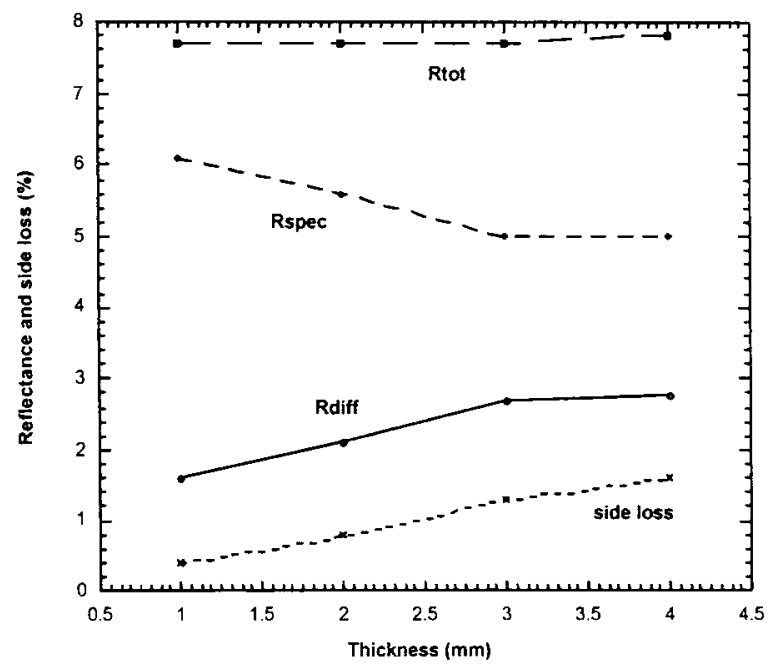

(a)

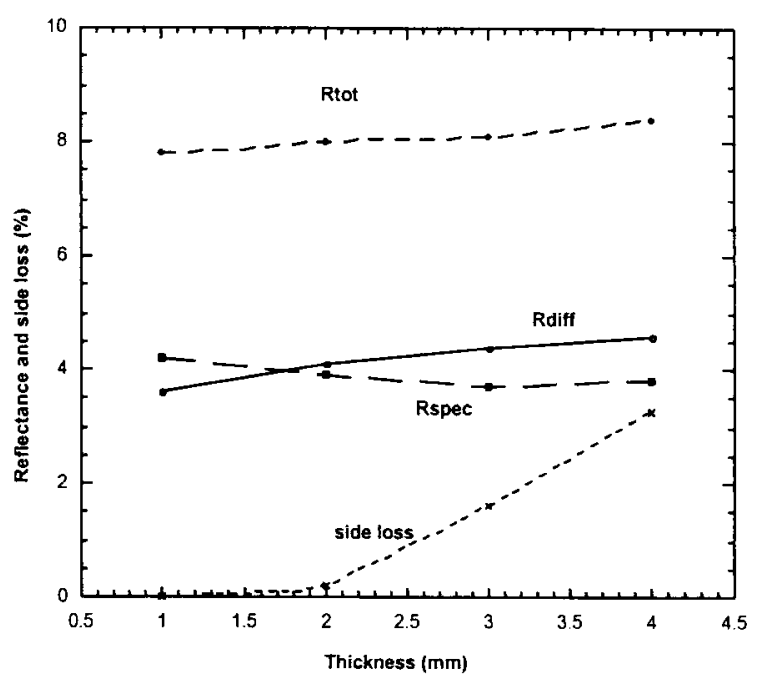

(b)

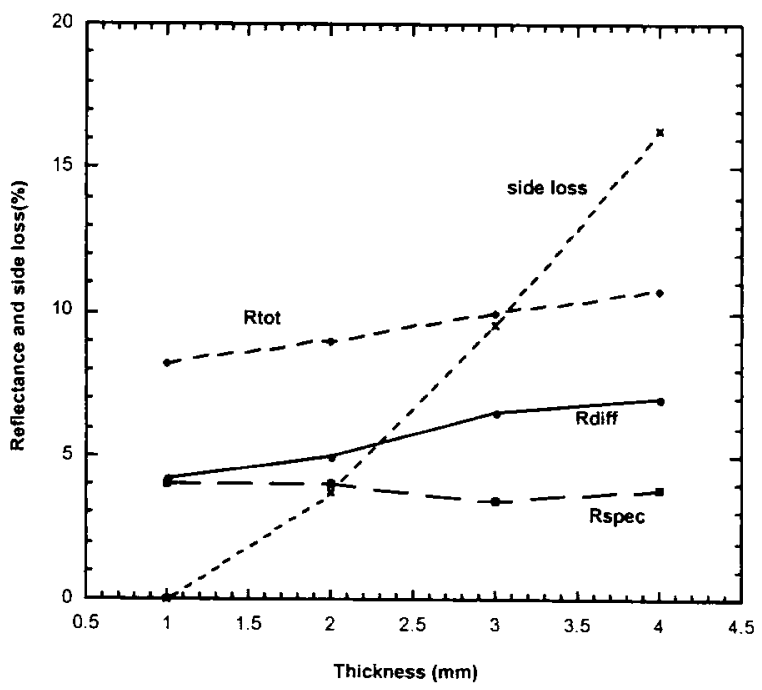

(c)

Fig. 10. Hemispherical total, diffuse, and specular reflectance and side loss at $520 \mathrm{~nm}$ as a function of thickness: (a) N80, (b) N73, (c) N77. 


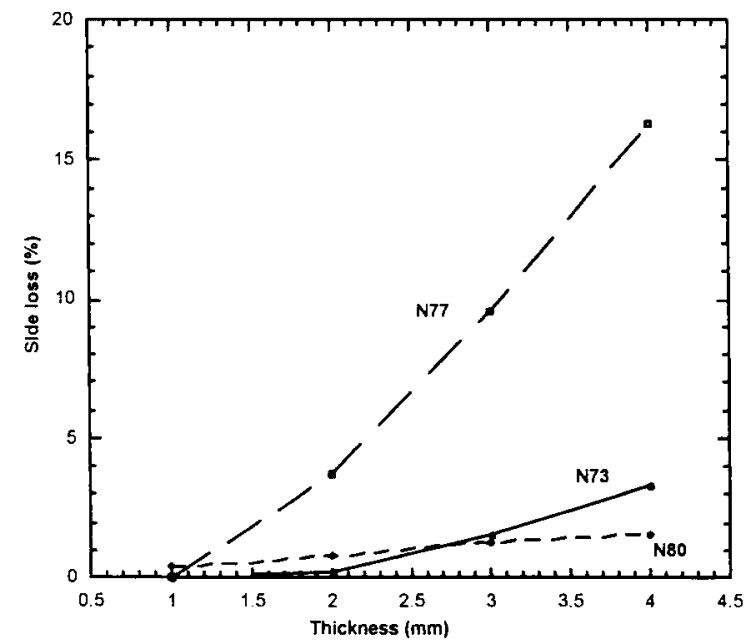

Fig. 11. Side loss as a function of thickness for each sample type.

This issue is still under investigation. At the moment, the $\mu$ value at any wavelength must be extracted from simulation modeling as described in Section 2 and fitted to light-spreading data. Experimental confirmation will require us to measure the particle indices directly across the spectrum of interest, for example, by mixing two different index liquids containing the particles, illuminating at different wavelengths, and measuring the mixed liquid indices where dispersion vanishes for each color.

Transmittance, as in Figs. 4-6, is the most pertinent to applications of these light diffusers. There is a continuous transition from dominance of specular transmittance in thin N80 to a dominance of diffuse transmittance in thick N77. There are also several important and interesting relationships among $\mathrm{d} T(\lambda) / \mathrm{d} \lambda$ and wavelength, sheet thickness, and particle concentration. These relationships appear to have different physical origins. Understanding these phenomena will enable a variety of controlled responses to be engineered.

Three distinct behavioral regimes can be identified for $\mathrm{d} T(\lambda) / \mathrm{d} \lambda$, and these are now discussed in detail.

\section{A. Spectral Regime A}

Both transmittance components have positive $\mathrm{d} T(\lambda) /$ $\mathrm{d} \lambda$. The 2-, 3-, and 4-mm N77 samples show this. It is noteworthy that they are the most strongly diffusing samples. The falloff in diffuse and specular $T$ values at shorter wavelengths in these three samples is linked directly to the strong side-loss spectra [Fig. 8(c)]. In contrast to this case, $\mathrm{d} T(\lambda) / \mathrm{d} \lambda$ are opposite in sign for diffuse and specular components in both regimes $\mathrm{B}$ and $\mathrm{C}$.

\section{B. Spectral Regime B}

Value $d T(\lambda / d \lambda$ is negative for the diffuse transmittance term and positive for the specular term. This intermediate regime is found in N73 at all thicknesses and in N77 for 1-mm thickness only. We attribute the difference between regime $\mathrm{A}$ and $\mathrm{B}$ to the transition from weak to strong side loss. Since the matrix components are common, we expect that internally (where we do not measure) both will have the same spectral dependence with a greater degree of light spreading and hence internal diffuse transmittance at shorter wavelengths compared with longer wavelengths. The interaction with the smooth exit surface brings about the different externally measured spectral responses. In regime $B$ nearly all the diffuse light exits from the rear surface. In these samples, internal light at $400 \mathrm{~nm}$ is more diffuse than internal light at $700 \mathrm{~nm}$. However, most rays across this spectral band are still not above the critical angle at the exit surface. Thus most exit and hence shorter wavelengths are recorded to be more diffuse. This contrasts with regime A where light striking the exit surface has been diffused over a much greater spread of angles than in regime B, with a big differential in spread at the blue wavelengths relative to the red wavelengths. In regime $\mathrm{A}$ there is strong TIR and hence side loss, which strongly depletes the exiting rays at shorter wavelengths relative to longer wavelengths. This strong internal differential diminishes the diffuse exit component at the blue relative to the red. Thus, in both the N77 and the N73 samples, shorter wavelengths undergo greater angular spreading than longer wavelengths at any given thickness. It is easy to misinterpret the experimental data by not taking the sideloss effect into account. There is a decrease in measured $T_{T}$ compared with true transmittance that is not due to absorption or reflection, but rather to the light-trapping effect that transports the light internally so that it is not collected by the measurement apparatus.

\section{Spectral Regime $\mathrm{C}$}

$\mathrm{d} T(\lambda) / \mathrm{d} \lambda$ is positive for the diffuse term whereas $\mathrm{d} T(\lambda) / \mathrm{d} \lambda$ is negative for the specular term. This occurs in N80 samples at all thicknesses, and because side loss is weak in this case it means that internal angular spread is larger at the red than the blue region of the spectrum. The N80 matrix material has a different molecular weight compared with the N73 and N77 samples, and the particles are also different from those used in these samples. Thus particle and host refractive indices will be different in N80 relative to N77 and N73. In the TRIMM system, the refractive indices of the matrix and particle are close, so small changes in the polymers can significantly alter scattering behavior. Thus we attribute regime $\mathrm{C}$ to a different wavelength dependence of the parameter $\mu$ that dictates scattering as noted in Section 2.

The observed reflectance behavior in the visible is unusual for a good diffuser and also warrants some comment. Total $R$ changes only slightly across the full range of specimens, from approximately 0.078 in all thicknesses of N80 (the exact value for this clear PMMA) to 0.11 in 4-mm N77. Two processes contribute to this change. First there is negligible backreflectance or scattering off the particles, so total reflectance is due almost entirely (except for N77) to 
Fresnel reflectance from the smooth back and front surfaces. The diffuse component of $R$ thus comes from light that has been reflected from the smooth back surface. In N80 only approximately half of this contributes to the diffuse component whereas half returns undeviated. However, in N73 nearly all of this, approximately $4 \%$ of the incident light, returns scattered. Finally, in N77 (with the highest particle concentration) the diffuse component increases above the expected Fresnel value of near 4\%. The rise in diffuse reflectance above $4 \%$ in N77 appears to be directly correlated with the side-loss term. That is, the few percent additional contributions to diffuse reflectance in N77 comes from light that is scattered through a large enough angle to undergo TIR at the back surface and is then deviated on the reverse path so that it falls below the critical angle. It then exits the front surface before it has traveled beyond the instrument's collection aperture.

Related to this is the other remarkable feature in these materials. It is the fact that most of the light trapped internally by TIR can propagate far enough so as not to contribute to the measurement; furthermore, much of the light gets right to the edges of these samples. Minimum direct path distances required for an edge exit in these samples is around $25 \mathrm{~mm}$. Actual ray paths would be much larger than these distances as they are traveling at oblique angles to the surface once they are above the critical angle. Actual paths to the side exit in the 4-mm sample where the effect is strongest in our data are above 40 $\mathrm{mm}$.

\section{Conclusion}

Clear polymer doped with TRIMM clear polymer particles can provide hemispherical transmittance close to that of a clear polymer sheet. Total hemispherical reflectance is also similar to that of a clear sheet. Spectrally these total values are quite flat in the visible range, except when side loss becomes strong. This is excellent for lighting research as the color rendering of lamps or sunlight will not be perturbed. The admix of diffuse and specular transmittance is controllable, as is the spectral variation of the diffuse component by use of changes in concentration, thickness, and variations within the polymer materials.

In analyzing optical data on materials such as these, it is necessary to account for light that is lost not by absorption but by TIR-induced trapping and then internal transport in the sheet beyond the collection aperture, and even to the sample's edges. The standard measurements of transmittance may thus underestimate the amount of light that is actually transmitted.

G. B. Smith is grateful to the Australian Research Council for both Large and Linkage grants that supported some of this research. J. C. Jonsson was able to visit the Department of Applied Physics at the University of Technology, Sydney, to perform parts of this research thanks to the J. Gust Richert Foundation. The contributions of research students Chris Deller and Alan Earp on other optical issues with these types of polymer contributed to our understanding. Werner Hoss and Klaus Albrecht of Roehm Degussa in Darmstadt kindly supplied the samples used in this study.

\section{References}

1. W. E. Vargas, "Diffuse radiation intensity propagating through a particulate slab," J. Opt. Soc. Am. A 16, 1362-1372 (1999).

2. G. B. Smith, A. Earp, J. Franklin, and G. McCredie, "Novel high performance scattering materials for use in energy saving light fittings and skylights based on polymer pigmented with polymer," in Solar and Switching Materials, C. M. Lampert, C.-G. Granqvist, and K. L. Lewis, eds., Proc. SPIE 4458, 10-18 (2001)

3. C. Deller, G. B. Smith, J. Franklin, and E. Joseph, "The integration of forward light transport and lateral illumination of polymer optical fiber," in Proceedings of the Australian Institute of Physics 15th Biannual National Congress (Australian Institute of Physics, Melbourne, 2002), pp. 307-309.

4. H. C. van de Hulst, Light Scattering by Small Particles (Dover New York, 1981).

5. C. M. Sorensen and D. J. Fischbach, "Patterns in Mie scattering," Opt. Commun. 173, 145-153 (2000).

6. J. A. Woollam, WVASE32 ellipsometer software (J. A. Woollam Co., Inc., Lincoln, Neb., 2001)

7. N. Lekishivili, L. Nadareishvili, G. Zaikov, and L. Khananashivili, "Polymers and polymeric materials for fiber and gradient optics," in New Concepts in Polymer Science, J. S. Vygodsky and S. A. Samsonya, eds. (VSP, BV, Utrecht, The Netherlands, 2002). 
THOMSON

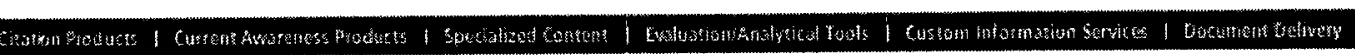

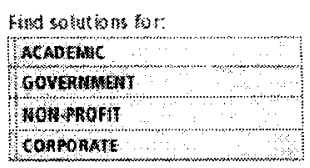

COAPORATE

\section{probitcas}

CONFEREACES AWO EVEWTS

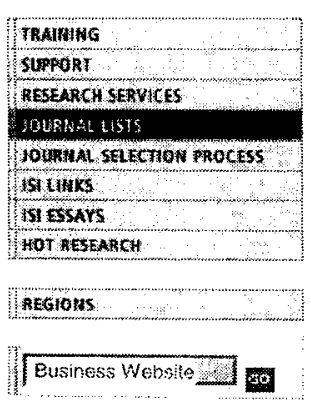

SEARCH RESULTS

IsI Master Journa list

SEARCH RESULTS

Searoh lems: $0003-6935$

Total oumels fouti:?

The following titiets; matched yo: renuest:

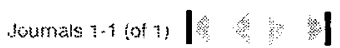

FOAMAT FOA PAIN!

APPLIED OPTCS

Biweekiy

1SS:1:0003.6335

OPTICAL SOC AMER, 2010 MASSACHUSEETTE AVE NW WASHMYGTON USA, DO, 20036

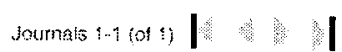

